\title{
AVALIAÇÃO DA ADIÇÃO DE SUCO DE ACEROLA EM SUCO DE ABACAXI VISANDO À PRODUÇÃO DE UM "BLEND" COM ALTO TEOR DE VITAMINA C ${ }^{1}$
}

\author{
FERNANDO CÉSAR AKIRA URBANO MATSUURA² e RENATA BERBERT ROLIM ${ }^{3}$
}

\begin{abstract}
RESUMO - O objetivo deste trabalho foi enriquecer em vitamina $\mathrm{C}$ o suco integral pasteurizado de abacaxi (SAb), através da adição de diferentes quantidades de suco integral pasteurizado de acerola (SAc) $(5 ; 10 ; 15$ e 20\%), produzindo um "blend" (mistura) destes sucos e visando à obtenção de um produto final que mantivesse as características sensoriais idênticas ao SAb original. As matérias-primas utilizadas foram polpas comerciais. Avaliação sensorial foi realizada dos atributos de cor, odor, consistência e sabor, através do teste de comparação múltipla. Análises físico-químicas de vitamina C (ácido ascórbico), sólidos solúveis totais, $\mathrm{pH}$ e acidez total titulável também foram feitas. Os resultados das análises sensoriais mostraram não existir diferença significativa $(\mathrm{p}>0,05)$ entre o $\mathrm{SAb}$ (padrão), o "blend" com 5\% de SAc e o "blend" com 10\% de SAc para todos os atributos avaliados nesse experimento, sendo que a adição de $10 \%$ de SAc resultou em um produto final com cerca de cinco vezes o teor de vitamina C do SAb original.
\end{abstract}

Termos para indexação: fruta, produto, ácido ascórbico.

\section{EVALUATION OF ACEROLA JUICE ADDITIONS TO PINEAPPLE JUICE FOR A HIGH VITAMIN C BLEND}

\begin{abstract}
The objective of this study was to enrich in vitamin $\mathrm{C}$ the integral pasteurized pineapple juice (PJ), by addition of different amounts of integral pasteurized acerola juice - $\mathrm{AJ}(5,10,15$ and $20 \%)$, to get a blend of these juices and a final product with the same sensorial characteristics as the PJ. The sources used were commercial pulps. Sensorial evaluation was carried out for colour, odour, consistence and flavour attributes, by using the multiple comparison test. Physical-chemical analyses of vitamin $\mathrm{C}$ (ascorbic acid), total soluble solids, $\mathrm{pH}$ and total titrable acidity were also done. The results of the sensorial analyses showed no significant difference $(p>0,05)$ between the PJ (pattern) and its blends with AJ at 5 and $10 \%$, for all attributes tested. The addition of AJ at $10 \%$ resulted in final product with about five times the vitamin $\mathrm{C}$ content of PJ.
\end{abstract}

Index terms: fruit, product, ascorbic acid.

\section{INTRODUÇÃO}

O hábito do consumo de sucos de frutas e hortaliças processados tem aumentado, motivado pela falta de tempo da população em preparar suco das frutas in natura, pela praticidade oferecida pelos produtos, substituição ao consumo de bebidas carbonatadas devido ao seu valor nutritivo e a preocupação com o consumo de alimentos mais saudáveis.

As frutas consistem em fonte nutricional de vitaminas, minerais e carboidratos solúveis, sendo que algumas possuem teor mais elevado de um ou de outro nutriente como, por exemplo, a acerola, que apresenta elevada quantidade de vitamina C. Outras frutas não são ricas no fornecimento de algum nutriente específico, como é o caso do abacaxi, que inclusive possui baixo teor de vitamina C (10 a $25 \mathrm{mg}$ de ácido ascórbico por $100 \mathrm{~g}$ de fruto); entretanto, apresentam grande aceitação sensorial por parte dos consumidores

O abacaxi é uma fruta cujo valor nutritivo se resume, praticamente, ao seu valor energético, devido a sua composição de açúcares. Os teores de proteína e de matéria graxa são inferiores a $0,5 \%$, sua contribuição como fonte de vitamina $\mathrm{C}$ é pequena em relação a outras fontes, e não apresenta, praticamente, nenhum outro nutriente em quantidade significativa. Merece destaque, todavia, o fato de que o abacaxi, pela sua atividade proteolítica, se constitui em coadjuvante da digestão dos alimentos, ao mesmo tempo que é matéria-prima para a extração da enzima bromelina, de larga aplicação na indústria de alimentos. Quanto à composição química, apresenta sólidos solúveis totais variando de 10,8 a $17,5^{\circ}$ Brix e acidez total titulável de 0,6 a $1,62 \%$ (expresso em ácido cítrico) (ITAL, 1987).

A acerola é uma fruta rica em vitamina $\mathrm{C}$, com teores de ácido ascórbico bastante variáveis ( 300 a $4.676 \mathrm{mg} / 100 \mathrm{~g}$ de polpa) (Mustard, 1946; Asenjo \& Moscoso, 1950; Jackson \& Pennock, 1958; Rocha, 1988; Itoo et al., 1990; Calvacante, 1991: Matsuura, 1994).

O suco de acerola pode ser usado vantajosamente como agente enriquecedor no processamento de numerosos sucos e néctares de frutos pobres em vitamina $\mathrm{C}$ (maçã, pêra, cereja, lima, abacaxi e pêssego) (Ledin, 1958). Nogueira (1991) também cita a possibilidade de uso do suco de acerola no enriquecimento de vitamina $\mathrm{C}$ de sucos e néctares.

Alguns estudos com "blends" de sucos de frutas

1 (Trabalho 038/2001). Recebido: 13/02/2001. Aceito para publicação: 01/02/2002.

2 Pesquisador, Tecnologia de Alimentos, Embrapa Mandioca e Fruticultura, Laboratório de Ciência e Tecnologia de Alimentos, Caixa Postal 007, 44380-000, Cruz das Almas, BA. T: (75) 6212120 / matsuura@cnpmf.embrapa.br

3 Estudante de graduação, Escola de Agronomia da Universidade Federal da Bahia (AGRUFBA), 44.380-000, Cruz das Almas, BA. T: (75) 6211220 
tropicais foram realizados, principalmente na formulação de néctares. Mostafa et al. (1997), em estudo com néctar de mamão com manga, observaram alta aceitação sensorial do produto formulado com $15 \%$ de polpa de mamão e $15 \%$ de polpa de manga. Salomon et al. (1977), em trabalhos com néctares de mamão com maracujá, concluíram que essas frutas apresentam condições excelentes para serem misturadas em diferentes proporções na elaboração de "blends". Estudos científicos do uso de suco de acerola na elaboração de "blends" com outras frutas tropicais ainda não foram realizados.

O objetivo principal deste trabalho foi aumentar a quantidade de vitamina $\mathrm{C}$ do suco de abacaxi, por meio da adição de quantidades variáveis de suco de acerola, esperando obter um "blend" que mantivesse as características sensoriais do suco de abacaxi original.

\section{MATERIAL E MÉTODOS}

\section{Material:}

Foram utilizados sucos integrais comerciais pasteurizados e congelados de abacaxi e acerola. Realizou-se mistura de suco integral pasteurizado de abacaxi com diferentes quantidades do suco integral pasteurizado de acerola $(5 \%, 10 \%$, $15 \%$ e $20 \%$ ), produzindo "blends" desses sucos. Os "blends" (produtos finais) preparados foram, logo em seguida, analisados, sendo que, para a análise sensorial, foram diluídos na proporção de 1:1 com água.

\section{Métodos:}

A análise sensorial foi realizada, avaliando os atributos de cor, odor, consistência e sabor dos produtos finais, através de teste de comparação múltipla (Moraes, 1988), utilizando-se de escala para comparação com o padrão (suco integral pasteurizado de abacaxi) com extremidades denominadas extremamente inferiores ao padrão (1) e extremamente melhores que o padrão (9), sendo igual ao padrão o valor 5 na escala (Figura 1).

Análises físico-químicas de $\mathrm{pH}$ (potenciômetro), sólidos solúveis totais (refratômetro, com correção de temperatura para $20^{\circ} \mathrm{C}$ ), acidez total titulável (método n.22.058 da AOAC, 1984) e ácido ascórbico (vitamina C) (método n43.064 da AOAC, 1984, modificado por Benassi, 1990) foram realizadas nas matériasprimas e nos produtos finais.

O delineamento experimental utilizado para a avaliação

Nome: Data:

Suco Pasteurizado de Abacaxi

Você recebeu uma amostra-padrão (P) e 05 amostras codificadas. Compare cada amostra com o padrão (se é MELHOR, IGUAL OU PIOR que o PADRÃO) em relação ao , avaliando o grau de diferença de acordo com a ESCALA abaixo.

1.Extremamente inferior ao padrão

2.Muito inferior ao padrão

3. Regularmente inferior ao padrão

4. Ligeiramente inferior ao padrão

5. Igual ao padrão
6. Ligeiramente melhor que o padrão

7. Regularmente melhor que o padrão

8. Muito melhor que o padrão

9. Extremamente melhor que o padrão
Valor
Número

da amostra

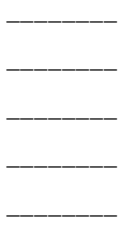

Comentários:

FIGURA 1 - Modelo da ficha utilizada na avaliação sensorial

sensorial correspondeu ao de blocos casualizados, utilizando 30 provadores não treinados, considerando-se 05 tratamentos e 03 repetições por tratamento. A análise estatística foi feita, empregando-se análise de variância, seguida de teste Dunnett para a comparação das médias dos tratamentos com o padrão (Gomes, 1973).

\section{RESULTADOS E DISCUSSÃO}

As análises físico-químicas das matérias-primas confirmaram o baixo conteúdo de vitamina $\mathrm{C}$ presente no suco integral pasteurizado de abacaxi (20,9 mg de ácido ascórbico por $100 \mathrm{~g}$ de suco) e o elevado conteúdo (1.000 mg de ácido ascórbico por $100 \mathrm{~g}$ de suco) no suco integral pasteurizado de acerola. $\mathrm{O}$ $\mathrm{pH}$, Brix e teor de acidez total titulável de ambos os sucos apresentaram valores considerados normais para o produto

TABELA 1 - Valores médios obtidos nas análises físico-químicas do suco integral pasteurizado de abacaxi (SAb) e de acerola (SAc) (matérias-primas).

$$
\text { Determinação }
$$

Valores*

\begin{tabular}{lcc}
\hline \multicolumn{1}{c}{ Determinação } & \multicolumn{2}{c}{ Valores* } \\
\cline { 2 - 3 } & \multicolumn{2}{c}{ S Ab } \\
\hline PH & $3,84 \pm 0,06$ & $3,37 \pm 0,05$ \\
Sólidos solúveis totais (\% a $\left.20^{\circ} \mathrm{C}\right)$ & $11,6 \pm 0,3$ & $8,0 \pm 0,2$ \\
Acidez total titulável $(\%$ de ácido) & $0,75 \pm 0,05^{* *}$ & $1,10 \pm 0,05^{* * *}$ \\
Vitamina C (mg de ácido ascórbico/100g de suco) & $20,9 \pm 1,0$ & $1.000,0 \pm 34,7$
\end{tabular}

*: Média e desvio-padrão de 03 amostras **: Expresso em ácido cítrico ***: Expresso em ácido málico 
TABELA 2 - Valores médios obtidos nas análises físico-químicas dos produtos finais ("blends") de suco integral pasteurizado de abacaxi, contendo diferentes quantidades de suco integral pasteurizado de acerola.

\begin{tabular}{ccccc}
\hline Tratamentos* & $\mathrm{pH}$ & $\begin{array}{c}\text { Sólidos solúveis } \\
\text { totais }(\%)^{* *}\end{array}$ & $\begin{array}{c}\text { Acidez } \\
(\%)^{* * *}\end{array}$ & $\begin{array}{c}\text { Ácido ascórbico } \\
(\mathrm{mg} / 100 \mathrm{~g})\end{array}$ \\
\hline $5 \%$ & $3,81 \pm 0,03$ & $11,2 \pm 0,2$ & $0,71 \pm 0,02$ & $79,3 \pm 3,7$ \\
$10 \%$ & $3,77 \pm 0,04$ & $11,0 \pm 0,2$ & $0,77 \pm 0,03$ & $115,4 \pm 5,1$ \\
$15 \%$ & $3,75 \pm 0,04$ & $10,9 \pm 0,1$ & $0,84 \pm 0,03$ & $182,7 \pm 6,6$ \\
$20 \%$ & $3,72 \pm 0,03$ & $10,6 \pm 0,2$ & $0,87 \pm 0,03$ & $245,2 \pm 6,2$ \\
\hline
\end{tabular}

Média e desvio-padrão de 03 amostras

* : Teor de suco integral pasteurizado de acerola no produto final ("blend")

** : Valores corrigidos para $20^{\circ} \mathrm{C} \quad * * *$ : Expresso em ácido cítrico

TABELA 3 - Valores médios obtidos na avaliação sensorial dos produtos finais ("blends") de suco integral pasteurizado de abacaxi, contendo diferentes quantidades de suco integral pasteurizado de acerola.

\begin{tabular}{ccccc}
\hline Tratamentos* & \multicolumn{4}{c}{ A tributos } \\
\cline { 2 - 5 } & C or & O dor & C onsistência & S abor \\
\hline Padrão & 5,0 & 4,7 & 4,6 & 4,9 \\
\hline $5 \%$ & $5,0 \mathrm{~N} \mathrm{~S}$ & $4,6 \mathrm{~N} \mathrm{~S}$ & $4,6 \mathrm{~N} \mathrm{~S}$ & $4,9 \mathrm{NS}$ \\
$10 \%$ & $4,8 \mathrm{~N} \mathrm{~S}$ & $4,2 \mathrm{~N} \mathrm{~S}$ & $4,6 \mathrm{~N} \mathrm{~S}$ & $4,3 \mathrm{NS}$ \\
$15 \%$ & $4,3 \mathrm{~N} \mathrm{~S}$ & $4,1 \mathrm{~N} \mathrm{~S}$ & $5,1 \mathrm{~N} \mathrm{~S}$ & $4,0 * *$ \\
$20 \%$ & $4,1 \mathrm{~N} \mathrm{~S}$ & $3,9 * *$ & $5,1 \mathrm{~N} \mathrm{~S}$ & 0,7 \\
\hline
\end{tabular}

* : Teor de suco integral pasteurizado de acerola no produto final ("blend")

Padrão - suco integral pasteurizado de abacaxi original

** : Tratamentos que diferiram do padrão, ao nível de 5\% de significância, pelo teste de Dunnet.

NS: Tratamentos que não diferiram do padrão, ao nível de 5\% de significância, pelo teste de Dunnet.

D.M.S. 5\%: diferença mínima significativa, ao nível de 5\%

(Tabela 1), concordantes com os valores citados para o abacaxi pelo ITAL (1987) e os encontrados para a acerola por Matsuura (1994).

Os produtos finais ("blends") com suco integral pasteurizado de abacaxi, contendo $5 \%, 10 \%, 15 \%$ e $20 \%$ de suco integral pasteurizado de acerola, mostram o elevado aumento do teor de vitamina $\mathrm{C}$ à medida que se aumentou a quantidade do suco de acerola no produto final, com teores chegando a quatro, seis, nove e doze vezes, respectivamente, ao encontrado no suco integral pasteurizado de abacaxi original (padrão e utilizado como matéria-prima) e com quantidades superiores ao de suco de laranja (teores médios na faixa de 40 a $70 \mathrm{mg} / 100 \mathrm{~g}$ de suco) que comumente é citado como fonte-padrão dessa vitamina. Em relação ao $\mathrm{pH}$, acidez total titulável e teor de sólidos solúveis totais, ocorreram decréscimos dos valores nos "blends" devido à maior acidez total titulável e menor teor de sólidos solúveis totais do suco integral pasteurizado de acerola (Tabela 2).

Os resultados da análise sensorial mostraram ocorrer diferença significativa $(\mathrm{p}<0,05)$ apenas nos tratamentos com $15 \%$ e $20 \%$, em relação ao atributo sabor, e no de $20 \%$, para o odor, em comparação ao padrão (Tabela 3). Ao se compararem as médias dos valores obtidos pelos tratamentos na escala sensorial apresentada para comparação múltipla pelos provadores (Figura 1), observa-se que: quanto ao atributo consistência, todos os tratamentos possuíram valores próximos a 5 (cinco) (termo "igual ao padrão" na escala); quanto ao atributo cor, os tratamentos com $5 \%$ e $10 \%$ possuíram valores próximos a 5 (cinco), e os tratamentos com $15 \%$ e $20 \%$, próximos a 4 (quatro) (termo "ligeiramente inferior ao padrão" na escala); quanto ao atributo sabor, o tratamento com $5 \%$ apresentou valor próximo a 5 (cinco), os tratamentos com $10 \%$ e $15 \%$, próximos a 4 (quatro) e o tratamento com $20 \%$, próximo a 3 (três) (termo "regularmente inferior ao padrão" na escala); quanto ao atributo odor, o tratamento com $5 \%$ apresentou valor próximo a 5 (cinco) e os tratamentos com $10 \%, 15 \%$ e $20 \%$, próximos a 4 (quatro) (Tabela 3). Portanto, o produto final ("blend") de suco integral pasteurizado de abacaxi contendo $5 \%$ ou $10 \%$ de suco integral pasteurizado de acerola manteve todas as características sensoriais avaliadas neste experimento, similares aos do suco integral pasteurizado de abacaxi original (padrão).

Nessa avaliação sensorial, verificou-se ser o atributo sensorial de sabor, o fator limitante na quantidade de suco integral pasteurizado de acerola possível de ser adicionada ao suco integral pasteurizado de abacaxi, seguido do atributo sensorial de odor. Os atributos sensoriais de consistência e cor não foram afetados pelas quantidades máximas adicionadas.

Estudos de estabilidade do produto devem ser realizados, a fim de avaliar a vida de prateleira dos mesmos. 


\section{CONCLUSÕES}

Os "blends" de suco integral pasteurizado do abacaxi, contendo $5 \%$ ou $10 \%$ de suco integral pasteurizado de acerola, apresentaram características sensoriais de odor, sabor, consistência e cor similares ao suco integral pasteurizado de abacaxi, com teor de vitamina $\mathrm{C}$ cerca de cinco vezes mais alto.

\section{REFERÊNCIAS BIBLIOGRÁFICAS}

A. O. A. C. (Associaton of Official Analytical Chemists). Official methods of analysis. 14. ed. Arlington: A.O.A.C., 1984. 1141p.

ASENJO C. F.; MOSCOSO, C. G. Ascorbic Acid Contet and other characteristics of West Indian Cherry. Food Research, Chicago, v. 15, p.103-106, 1950.

BENASSI, M. T. Análise dos efeitos de diferentes parâmetros na estabilidade de vitamina $C$ em vegetais processados. Campinas, 1990. 159p. Dissertação (Mestrado em Ciência de Alimentos), Faculdade de Engenharia de Alimentos, Universidade Estadual de Campinas.

CAVALCANTE, M. L. Composição de carotenóides e valor de vitamina A na pitanga (Eugênia uniflora) e acerola (Malpighia glabra L.). Rio de Janeiro, 1991. 86p. Dissertação (Mestrado em Nutrição), Instituto de Nutrição, Universidade Federal do Rio de Janeiro.

GOMES, F. P. Curso de Estastística Experimental 5. ed. São Paulo: Nobel, 1973. 468p.

ITAL Abacaxi: cultura, matéria-prima, processamento e aspectos econômicos. 2. ed. Campinas, SP: ITAL, 1987. 285p.

ITOO, S. ; AIBA, M. ; ISHIHATA, K. Comparison of ascorbic acid content in acerola fruit from different production region depend on degree of maturity, and it's stability by pocessing. Nipon Shokuhim Kogyo Gakkaishi, Tóquio, v.37, n.9, p.726-
729,1990

JACKSON, G. C. PENNOCK, N. Fruit and vitamin C production of five and six-year-old acerola trees. Journal of Agriculture of the University of Puerto Rico, Rio Piedras, v. 42, p.196-205, 1958.

LEDIN, R. B. A comparison of three clones of Barbados Cherry and the importance of improved selections for commercial plantings. The Proceedings of Florida State Horticultural Society, Goldenrod, v. 69, p. 293-297, 1956.

MATSUURA, F. C. A. U. Processamento e caracterização de suco integral concentrado congelado de acerola. Campinas, 1994. 141p. Dissertação (Mestrado em Tecnologia de Alimentos), Faculdade de Engenharia de Alimentos, Universidade Estadual de Campinas.

MORAES, M. A. C. Métodos para a avaliação sensorial dos alimentos. 7.ed. Campinas, SP: Ed. da Unicamp, 1988. 93.

MOSTAFA, G. A.; ABD-EL-HADY, E. A.; ASKAR, A. Preparation of papaya and mango nectar blends. Fruit Processing, Chicago, v.7, n.5, p. 180-185, 1997.

MUSTARD, M. J. The ascorbic acid content of same Malpighian fruit and jellys. Science, Washington, v.104, p. 230-235, 1946.

NOGUEIRA, C. M. C. da C. D. Estudo químico e tecnológico da acerola (Malpighia glabra L.). Fortaleza, 1991. 117p. Dissertação (Mestrado em Ciências), Universidade Federal do Ceará.

ROCHA, I. C. Suco de Acerola: Efeito da temperatura de pasteurização e armazenamento. Recife, 1988. 105p. Dissertação (Mestrado em Ciências da Saúde). Centro de Ciências da Saúde, Universidade Federal do Pernambuco.

SALOMON, E. A. G.; KATO, K; MARTIN, Z. J. de; SILVA, S. D. da; MORI, E. E. M. Estudo das composições (blending) do néctar de mamão-maracujá. Boletim do Instituto de Tecnologia de Alimentos, Campinas, n.51, p. 165-179, 1977. 